\title{
Validation of the English Severe Respiratory Insufficiency Questionnaire
}

\author{
Dipansu Ghosh*, Peter Rzehak ${ }^{\#,{ }^{\ddagger},+}$, Mark W. Elliott* and Wolfram Windisch ${ }^{\S}$
}

\begin{abstract}
Assessment of health-related quality of life in patients with respiratory failure on home ventilation requires appropriate and highly specific measurement tools. We attempt to validate the English version of the Severe Respiratory Insufficiency Questionnaire (SRI).

Psychometric properties of the SRI in 152 patients established on home ventilation were assessed.
\end{abstract}

Cronbach's alpha ranged between 0.77 and 0.89 for the seven subscales and and was 0.93 for the summary scale. Principal components analysis revealed a one-factor solution for four and two factors for three subscales. Confirmatory factor analysis revealed a two-factor solution for six subscales, but these factors were dependent on each other. One factor was extracted out of the subscales confirming one summary scale accounting for $70 \%$ of the total variance. Correlation analysis between scales of the SRI and the Medical Outcome Study 36-item short-form health survey demonstrated highest correlations between comparable subscales. Chronic obstructive pulmonary disease patients had lower summary scale scores than patients with restrictive chest wall diseases, neuromuscular disorders and obesity hypoventilation syndrome.

The English SRI has high internal consistency reliability, clearly established construct and concurrent validity, and is capable of differentiating between different diseases. It is now validated for use in research involving patients receiving home ventilation.

KEYWORDS: Assisted ventilation, noninvasive ventilation, quality of life, reliability and validity, respiratory insufficiency

$\mathrm{H}$ ealth-related quality of life (HRQL) questionnaires have become an essential part of managing patients with chronic conditions to assess the impact of these conditions and the effect of interventions on daily life [1,2]. Over the past few decades several generic and disease-specific questionnaires have been developed. These are multidimensional tools to assess several aspects of patients' lives that are otherwise not objectively studied during clinical care. Generic questionnaires include the Sickness Impact Profile and the Medical Outcome Study (MOS) 36-item Short-form Health Survey (SF-36), which are not specific to any particular disease and are therefore most commonly used for general survey research, and allow comparisons between different diseases [3, 4]. In contrast, disease-specific questionnaires focus on domains most relevant to a particular disease; for patients with chronic respiratory disease the Chronic Respiratory Disease Questionnaire (CRQ) and the St George's Respiratory Questionnaire (SGRQ) are often used. These questionnaires are well validated for patients suffering from chronic obstructive pulmonary disease (COPD) [5, 6]. However, until recently, no specific questionnaires have been available for patients with chronic respiratory failure, particularly those requiring chronic ventilatory support and receiving home mechanical ventilation (HMV). Although these patients may have a variety of diseases and comorbidities, chronic severe respiratory failure is common to all [7]. Many important aspects of these patients' lives are not covered by generic or COPD-specific questionnaires, and this results in an incomplete assessment of HRQL [8-10]. Therefore, a questionnaire specifically designed for this patient group would be desirable.

The Severe Respiratory Insufficiency Questionnaire (SRI) is a recently developed multidimensional HRQL questionnaire found to have good psychometric properties when used in the population it was specifically designed for, namely patients receiving $\mathrm{HMV}$, and has been shown to be sensitive to change [11-16]. This questionnaire, originally
AFFILIATIONS

*Dept of Respiratory Medicine, St James's University Hospital, Leeds UK.

\#Helmholtz Zentrum München, German Research Center for Environmental Health, Institute of Epidemiology, Neuherberg, •Ludwig-Maximilians University Munich, Institute of Medical Data Management, Biometrics and Epidemiology,

${ }^{+}$Division of Metabolic and Nutritional Medicine, Dr von Hauner Children's Hospital, Ludwig-Maximilians

University of Munich Medical Centre, Munich, and

${ }^{\S}$ Clinic of Cologne, University of Witten/Herdecke, Dept of Pneumology, Cologne, Germany.

CORRESPONDENCE

W. Windisch

Clinic of Cologne

University of Witten/Herdecke

Dept of Pneumology

Ostmerheimer Strasse 200

D - 51109 Köln

Germany

E-mail: windischw@kliniken-

koeln.de

Received:

Sept 052011

Accepted after revision:

Nov 282011

First published online:

Dec 192011 
developed in German, has recently been professionally translated into several languages, including Danish, Dutch, French, Greek, Hebrew, Japanese, Norwegian, Polish, Spanish and Swedish. Data on the translations process, validation and clinical application have already been published for the Dutch and Spanish versions [17-20]. A French validation is under progress. However, an English version has not yet been provided. Therefore, the aim of the present study was to create and validate an English version of the SRI. Preliminary data of this study has been published as an abstract [21].

\section{METHODS}

\section{Study design and population}

This cross-sectional study was conducted in the Dept of Respiratory Medicine at St James' University Hospital, Leeds, UK, a tertiary care teaching hospital. Ethical approval was obtained from the local National Health Service ethics committee. Patients with chronic hypercapnic respiratory failure from a wide variety of causes established on HMV for at least 1 month were eligible for the study. They had to be in a clinically stable state, without any changes of medication during the previous 4 weeks. Patients with evidence of acute respiratory failure, i.e. patients with worsening of symptoms during the previous 2 weeks, a $\mathrm{pH}<7.35$, or with signs of respiratory infection (two of the following: coloured sputum, fever, infiltration on chest radiography, white blood cell count $>10,000 \mu \mathrm{L}^{-1}$ ) were excluded. Patients received noninvasive ventilation (NIV) or invasive ventilation via tracheostomy. Arterial blood gas samples were taken during visits to the outpatient clinic and not when on ventilation, unless patients were ventilatordependent (e.g. tracheostomised).

Each patient completed the SRI and the MOS SF-36 in their own time $[4,11]$. Both questionnaires were provided at the same time. The underlying diagnoses leading to respiratory failure were recorded; in cases in which there was more than one condition contributing to respiratory failure the one deemed to be contributing most was used for categorisation purposes. Patients were categorised into five categories: COPD, restrictive chest wall disorders (RCWD), neuromuscular disorders (NMD), obesity hypoventilation syndrome (OHS) and "miscellaneous".

\section{The SRI questionnaire and the MOS SF-36}

The English SRI is the result of transcultural adaptation [1, 2]. This includes translation and back-translation of the original German SRI by two independent professional translators. Items with incongruence or discrepancies between the original and back-translated version have been accordingly modified in the definitive UK English version. The details of the adaptation process are described in the online supplement.

The SRI consists of seven subscales covering 49 items: respiratory complaints (SRI-RC), physical functioning (SRI$\mathrm{PF}$ ), attendant symptoms and sleep (SRI-AS), social relationships (SRI-SR), anxiety (SRI-AX), psychological well-being (SRI-WB) and social functioning (SRI-SF). These seven subscales can be summarised to one summary scale (SRI-SS). Rating for each item provided by a five-point Likert scale with scores ranging between 0 and 100 for every scale is possible following transformation of raw values, as previously described [11]. Higher scores for this tool are attributed to better HRQL.

The SF-36 consists of eight subscales measuring different aspects of health status with lower scores (0-100) indicating poorer health or higher disability: SF-36-PF: physical functioning; SF-36-RP: role-physical; SF-36-BP: bodily pain; SF-36-GH: general health; SF-36-VT: vitality; SF-36-SF: social functioning; SF-36-RE: role-emotional; SF-36-MH: mental health [4, 22].

\section{Statistical analysis}

Reliability of each subscale was calculated as internal consistency of response among the items assigned to each scale by Cronbach's alpha [23]. Values above 0.8 indicate high and above 0.9 very high correlations among the items of a scale and thus may be interpreted as measuring the same construct. Construct validity was assessed by both explorative factor analysis using principal components analysis (PCA) and confirmatory factor analysis (CFA). For PCA, only factors with eigenvalues higher than unity (1) have been extracted, i.e. the standardised variance of at least one item variable of the scale

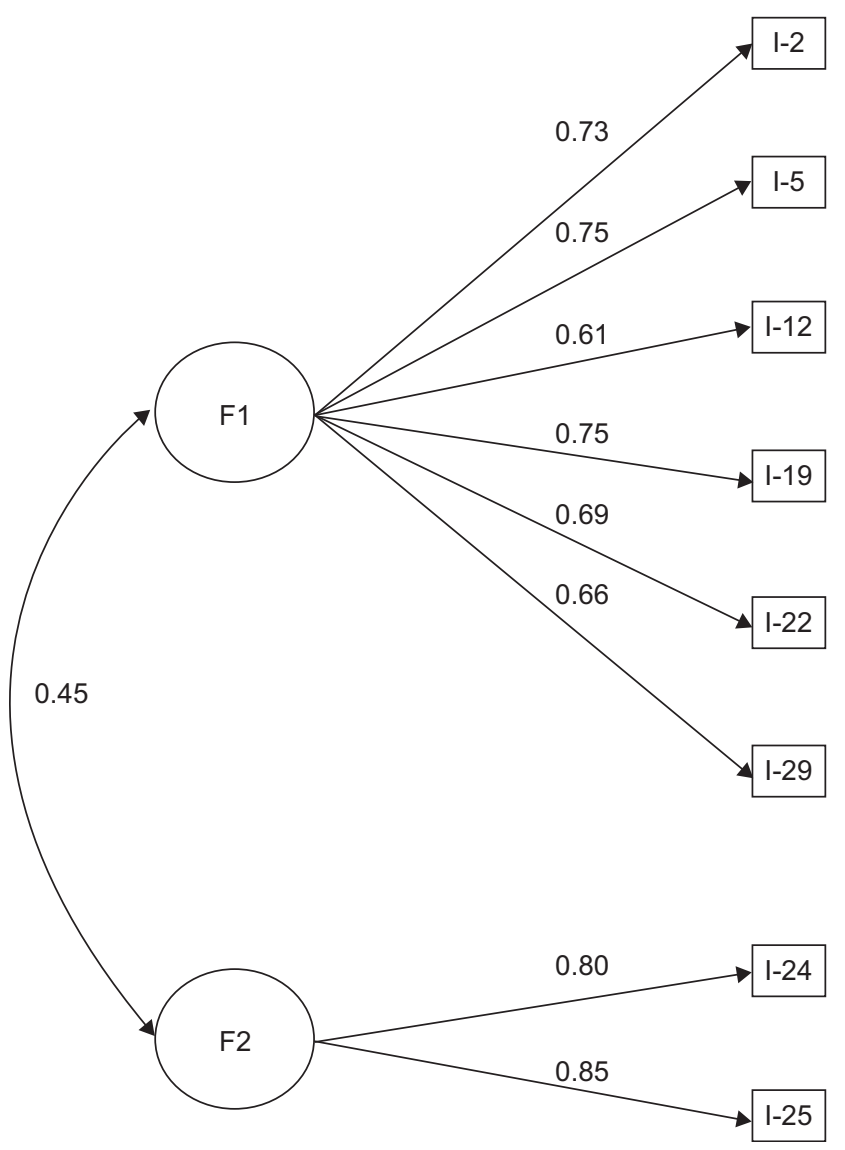

FIGURE 1. Visual illustration of final two-factor model of scale respiratory complaints reported in table 6. Ovals indicate latent factors F1 and F2, which are regressed on the observed variables (items) indicated by boxes (with the respective item number) in the confirmatory factor analysis model (CFA). The value near the double-headed arrow is the correlation between the two factors F1 and F2. The values near the single-headed arrows are the factor loadings (or regression coefficients) standardised to both the latent factor and observed variables' variances of the CFA model. 
TABLE 1 Values for use of noninvasive ventilation (NIV), lung function and blood gases corresponding to the underlying disease

\begin{tabular}{|c|c|c|c|c|c|c|}
\hline Categories & COPD & RCWD & NMD & OHS & Miscellaneous & All \\
\hline Patients & $26(17)$ & $58(38)$ & $32(21)$ & $29(19)$ & 7 (5) & 152 \\
\hline Duration of NIV months & $46.9 \pm 25.6$ & $70.7 \pm 47.3$ & $64.6 \pm 46.9$ & $50.4 \pm 25.9$ & $48.3 \pm 42.2$ & $60.4 \pm 41.2$ \\
\hline Average use per night $h$ & $6.8 \pm 1.5$ & $8.0 \pm 2.4$ & $11.2 \pm 6.2$ & $7.4 \pm 1.8$ & $8.9 \pm 3.7$ & $8.4 \pm 3.8$ \\
\hline FEV 1 L & $0.9 \pm 0.5$ & $0.7 \pm 0.4$ & $1.1 \pm 0.5$ & $1.4 \pm 0.7$ & & $1.0 \pm 0.6$ \\
\hline pH & $7.40 \pm 0.04$ & $7.41 \pm 0.04$ & $7.40 \pm 0.04$ & $7.38 \pm 0.03$ & & $7.41 \pm 0.04$ \\
\hline $\mathrm{PCO}_{2} \mathrm{kPa}$ & $6.9 \pm 1.3$ & $6.5 \pm 1.4$ & $6.1 \pm 1.6$ & $6.3 \pm 1.0$ & & $6.4 \pm 1.4$ \\
\hline $\mathrm{PO}_{2} \mathrm{kPa}$ & $8.6 \pm 1.5$ & $9.2 \pm 1.6$ & $10.5 \pm 2.1$ & $9.5 \pm 2.9$ & & $9.4 \pm 2.0$ \\
\hline $\mathrm{HCO}_{3} \mathrm{mmol} \cdot \mathrm{L}^{-1}$ & $31 \pm 7.4$ & $29.3 \pm 4.1$ & $26.6 \pm 3.9$ & $26.9 \pm 2.4$ & & $28.5 \pm 4.5$ \\
\hline SRI-SS & $43.1 \pm 17.3$ & $61.9 \pm 16.1$ & $58.8 \pm 20.3$ & $53.4 \pm 18.8$ & $53.5 \pm 19.7$ & $55.9 \pm 18.9$ \\
\hline
\end{tabular}

Data are presented as $n(\%)$ or mean \pm SD. COPD: chronic obstructive pulmonary disease; RCWD: restrictive chest wall disorders; NMD: neuromuscular disorders; OHS: obesity hypoventilation syndrome; BMI: body mass index; FEV1: forced expiratory volume in $1 \mathrm{~s}$; FVC: forced vital capacity; $\mathrm{PCO}_{2}$ : carbon dioxide tension; $\mathrm{PO}_{2}$ : oxygen tension; SRI-SS: Severe Respiratory Insufficiency Questionnaire summary scale.

is accounted for by the common factor. In simple terms, factors with eigenvalue $>1$ are considered to be significant, explaining an important amount of variability in the data.

In applying CFA we hypothesised in the first step that the observed correlations among specific items are the result of the effect of one common (latent) factor or construct for each of the eight SRI scales (e.g. the six items for scale SRI-PF), and that therefore these items can be combined to a common scale. Whether each of these postulated one-factor models hold analysing the data was evaluated by several model-fit indexes described below. If the one-factor solution was rejected a twocommon-factor model was estimated with items reassigned to two (potentially correlated) factors informed by the substantial meaning of the items and by so-called modification indexes (MI) of the software programme, indicating potential improvements of fit when respecifying the CFA model accordingly [24]. The fit for a model with pre-specified common factors was evaluated by the comparative fit index (CFI) and by testing that root mean square error of approximation (RMSEA) is $<0.05$. CFI values $\geqslant 0.95$ and RMSEA $p$-values $>0.05$ indicate a good overall fit of the factor model. A visual illustration of the final two-factor CFA model of the SRI-RC scale is depicted in figure 1 and for all other scales in the online supplement.

Group comparisons on the SRI between patients of different underlying diagnosis were performed using ANOVA with Tukey-Kramer corrections for multiple comparisons. A p-value of $<0.05$ was considered to be statistically significant.

Further information on methodology, detailed statistical analysis and the English SRI is provided in the online data supplement.

\section{RESULTS}

A total of 152 patients (75 females) completed the questionnaires (table 1) with 142 and 10 patients receiving NIV and invasive (tracheostomy) ventilation, respectively. Patients with NMD were younger $(p<0.0001)$ and had the highest overnight NIV use $(\mathrm{p}<0.0001)$ when compared to COPD, RCWD and OHS patients. There were no differences amongst the other three groups (COPD, RCWD and OHS).

TABLE 2 Distribution of patients under different categories not suffering from chronic obstructive pulmonary disease (COPD) or obesity hypoventilation syndrome

\begin{tabular}{|c|c|c|c|}
\hline Restrictive chest wall disorders & Neuromuscular conditions & Miscellaneous & Tracheostomised patients \\
\hline Idiopathic kyphoscoliosis: 33 & Muscular dystrophy: 11 & Central sleep apnoea: 4 & Prader-Willi syndrome: 1 \\
\hline Post-tubercular sequelae: 4 & Myopathy: 6 & Cystic fibrosis: 2 & Muscular dystrophy: 2 \\
\hline Post-polio kyphoscoliosis: 10 & Myotonic dystrophy: 3 & Brain stem mass: 1 & Central sleep apnoea: 1 \\
\hline Other kyphoscoliosis: 4 & Motor neurone disease: 3 & & Motor neurone disease: 1 \\
\hline \multirow[t]{2}{*}{ Thoracoplasty: 7} & Spinal muscular atrophy: 4 & & Myasthenia gravis: 1 \\
\hline & & & Spinal cord injury: 2 \\
\hline Total: $\mathbf{5 8}$ & Total: $\mathbf{3 3}$ & Total: 7 & Total: 10 \\
\hline
\end{tabular}




\begin{tabular}{|c|c|c|c|c|c|c|c|}
\hline \multirow{2}{*}{$\begin{array}{l}\text { TABLE } 3 \\
\text { Scale }\end{array}$} & \multicolumn{7}{|c|}{$\begin{array}{l}\text { Descriptive data of the } 36 \text {-item Short-form Health } \\
\text { Survey (SF-36) and the Severe Respiratory } \\
\text { Insufficiency Questionnaire (SRI) }\end{array}$} \\
\hline & n & Mean \pm SD & P25 & P50 & P75 & Min. & Max. \\
\hline SF-36-PF & 143 & $20.0 \pm 22.9$ & 0 & 15 & 30 & 0 & 95 \\
\hline SF-36-RP & 137 & $31.8 \pm 39.0$ & 0 & 0 & 50 & 0 & 100 \\
\hline SF-36-BP & 144 & $56.2 \pm 29.7$ & 31 & 52 & 84 & 0 & 100 \\
\hline SF-36-GH & 143 & $33.5 \pm 23.2$ & 15 & 30 & 50 & 0 & 100 \\
\hline SF-36-VT & 144 & $41.3 \pm 23.5$ & 20 & 43 & 55 & 0 & 100 \\
\hline SF-36-SF & 148 & $58.6 \pm 30.6$ & 38 & 63 & 88 & 0 & 100 \\
\hline SF-36-RE & 136 & $59.6 \pm 44.2$ & 0 & 67 & 100 & 0 & 100 \\
\hline SF-36-MH & 144 & $70.9 \pm 20.4$ & 58 & 76 & 88 & 16 & 100 \\
\hline SRI-RC & 149 & $52.7 \pm 20.9$ & 38 & 53 & 67 & 3 & 97 \\
\hline SRI-PF & 148 & $42.2 \pm 22.3$ & 25 & 42 & 58 & 0 & 96 \\
\hline SRI-AS & 148 & $56.8 \pm 20.5$ & 43 & 57 & 71 & 4 & 100 \\
\hline SRI-SR & 150 & $70.6 \pm 21.4$ & 58 & 71 & 88 & 0 & 100 \\
\hline SRI-AX & 149 & $52.3 \pm 27.2$ & 35 & 55 & 75 & 0 & 100 \\
\hline SRI-WB & 147 & $59.5 \pm 21.3$ & 44 & 61 & 75 & 0 & 100 \\
\hline SRI-SF & 149 & $60.6 \pm 24.4$ & 41 & 57 & 84 & 6 & 100 \\
\hline SRI-SS & 141 & $55.9 \pm 18.9$ & 41 & 56 & 71 & 5 & 97 \\
\hline
\end{tabular}

P25, P50 and P75: 25, 50 and 75th percentiles. SF-36 scales: SF-36-PF: physical functioning; SF-36-RP: role-physical; SF-36-BP: bodily pain; SF-36GH: general health; SF-36-VT: vitality; SF-36-SF: social functioning; SF-36-RE: role-emotional; SF-36-MH: mental health. SRI scales: SRI-RC: respiratory complaints; SRI-PF: physical functioning; SRI-AS: attendant symptoms and sleep; SRI-SR: social relationships; SRI-AX: anxiety; SRI-WB: psychological well-being; SRI-SF: social functioning; SRI-SS: summary scale.

Table 2 shows the distribution of the different conditions leading to chronic respiratory failure. Of the 29 patients with OHS, five patients had lone OHS; seven patients also had COPD; 15 patients had combined obstructive sleep apnoea and OHS; one patient also had scoliosis and one patient had PraderWilli syndrome. 31 patients were receiving long-term oxygen therapy in addition to HMV; 10 patients each in the COPD, RCWD and OHS groups, and one patient with cystic fibrosis.

Descriptive data of both the SRI and the SF-36 questionnaires are provided in table 3 . Five of the eight scales (subscales and summary scale) were normally distributed; in three subscales (SRI-SR, SRI-AX and SRI-SF) normality was formally not achieved. However, the distribution of all SRI scales was rather homogenous as shown by the percentiles given in table 3 . Thereby the SRI covered a broad range of the questionnaire's scaling range. The mean score of the SRI-SS $(55.9 \pm 18.9)$ was roughly in the middle of the questionnaire's scaling range, which was also true for most of the subscales (table 3). In contrast, none of the SF-36 scales was normally distributed, and the distribution was severely heterogeneous. This was particularly true for SF-36-PF, SF-36-RP and SF-36-GH, where most patients demonstrated the most severely reduced scores (median ranging between 0 and 30 ).

\section{Reliability}

Cronbach's alpha reliability ranged between 0.77 and 0.89 for the seven subscales (table 4). Compared to the reliability of the

\begin{tabular}{|c|c|c|c|c|}
\hline \multirow{2}{*}{$\begin{array}{l}\text { TABLE } 4 \\
\text { Scale }\end{array}$} & \multicolumn{4}{|c|}{$\begin{array}{l}\text { Scale means and reliability of the Severe } \\
\text { Respiratory Insufficiency Questionnaire (SRI) } \\
\text { scales }\end{array}$} \\
\hline & Items & Patients & Scores & Cronbach's $\alpha$ \\
\hline SRI-RC & 8 & 149 & $52.7 \pm 20.9$ & 0.84 \\
\hline SRI-PF & 6 & 148 & $42.2 \pm 22.3$ & 0.77 \\
\hline SRI-AS & 7 & 148 & $56.8 \pm 20.5$ & 0.81 \\
\hline SRI-SR & 6 & 150 & $70.6 \pm 21.4$ & 0.81 \\
\hline SRI-AX & 9 & 149 & $52.3 \pm 27.2$ & 0.87 \\
\hline SRI-WB & 5 & 147 & $59.5 \pm 21.3$ & 0.89 \\
\hline SRI-SF & 8 & 149 & $60.6 \pm 24.4$ & 0.87 \\
\hline SRI-SS & 7 scales & 141 & $55.9 \pm 18.9$ & 0.93 \\
\hline \multicolumn{5}{|c|}{$\begin{array}{l}\text { Data are presented as } n \text { or mean } \pm \text { SD, unless otherwise stated. SRI-RC: } \\
\text { respiratory complaints; SRI-PF: physical functioning; SRI-AS: attendant } \\
\text { symptoms and sleep; SRI-SR: social relationships; SRI-AX: anxiety; SRI-WB: } \\
\text { psychological well-being; SRI-SF: social functioning; SRI-SS: summary scale. }\end{array}$} \\
\hline
\end{tabular}

subscales, the reliability of the summary scale was even higher (0.93; table 4).

\section{Validity}

PCA of the subscale-related items revealed that by applying the eigenvalue criterion of $\geqslant 1$ for factor extraction a one-factor solution is sufficient for four subscales (uni-dimensional construct), but that a two-factor solution (two-dimensional construct) is required for three subscales (table 5). In addition, only one factor with an eigenvalue higher than unity could be extracted out of the seven subscales for the summary scale (uni-dimensional construct). This factor accounted for $70 \%$ of the total variance, thus confirming one single summary scale pooling of the seven subscales.

Further analysis of the seven subscales using the CFA demonstrated a consistent two-factor solution for six of the seven subscales (all but SRI-SF; table 6). As an example, one factor of the subscale SRI-AS covered non-respiratory daytime symptoms (F1; table 6) and the other factor covered nocturnal symptoms (F2; table 6). The model fit as estimated from the CFI was excellent $(\geqslant 0.95)$ for all subscales. The model fit as evaluated by the p-value of the RMSEA test, that the RMESA is less than 0.05, was also sufficient for all but one scale (SRI-AX). However, both factors in the six subscales with a two-factor solution were substantially correlated ( $r$ range $0.45-0.92)$ indicating that both factors were not independent from each other (table 6 and fig. 1).

In addition, a significant concurrent validity was confirmed by the correlation analysis between scales of the SRI and scales of the SF-36 (table 7). The highest correlations were found between SRI-SF and SF-36-SF $(r=0.8, \mathrm{p}<0.001)$ and SRI-WB and SF-36-MH $(r=0.78, \mathrm{p}<0.001)$. The lowest correlation was found between SRI-SR and SF-36-PF $(r=0.14 ; \mathrm{p}=0.09)$. Overall, correlations were high when subscales of the SRI and SF-36 focused on comparables aspects of HRQL (e.g. both subscales addressing physical, emotional or social aspects), but was lower when subscales targeting different aspects were correlated (table 7). 


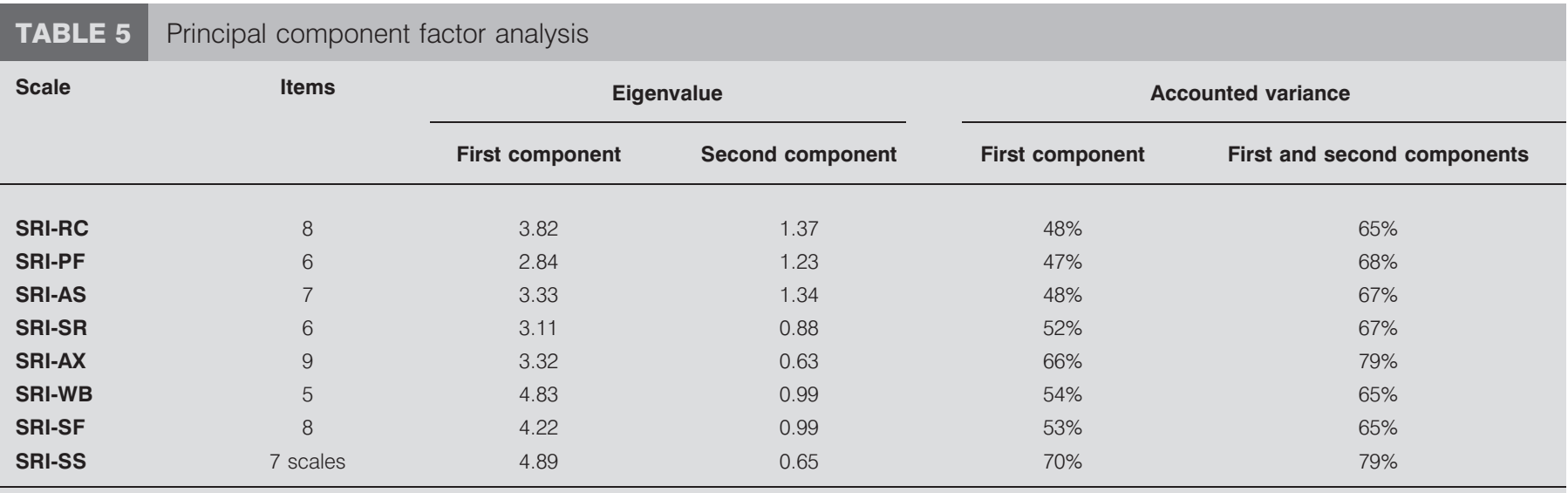

SRI: Severe Respiratory Insufficiency Questionnaire; SRI-RC: respiratory complaints; SRI-PF: physical functioning; SRI-AS: attendant symptoms and sleep; SRI-SR: social relationships; SRI-AX: anxiety; SRI-WB: psychological well-being; SRI-SF: social functioning; SRI-SS: summary scale.

\section{Disease comparisons}

Overall, there were significant $(\mathrm{p}=0.0008)$ differences between different diagnostic groups (COPD, RCWD, NMD, OHS and miscellaneous) for the SRI-SS (table 1). When comparing single diagnostic groups, COPD patients had lower SRI-SS scores than patients with RCWD ( $p<0.0001)$ NMD $(p=0.0016)$ and OHS $(p=0.0387)$ after adjustment for multiple comparisons, but there were no significant differences when comparing nonCOPD patients.

COPD patients also had significantly lower sores $(p<0.05)$ compared to RCWD patients in all SRI-subscales except SRI$\mathrm{SR}$, which was, however, close to statistical significance $(p=0.06)$. In addition, COPD patients also had lower scores $(\mathrm{p}<0.05)$ compared to NMD patients in three of the seven subscales (SRI-RC, SRI-AX and SRI-SF).

\section{DISCUSSION}

The SRI is a disease-specific questionnaire with established psychometric properties, which is currently becoming an international standard tool to assess HRQL in patients with severe chronic respiratory failure. The current study establishes that the English SRI, which resulted from professional translation and back-translation of the original German version, has high psychometric properties. Therefore, the English SRI qualifies for being used in clinical studies assessing HRQL in patients with severe chronic respiratory failure. The main findings gained by the present study can be summarised as follows.

The internal consistency as shown by Cronbach's alpha was higher than 0.8 for all subscales except for one (SRI-PF), which, however, was 0.77 and therefore considerably higher than the value of 0.7 considered to be sufficiently reliable for use in group comparisons [23]. Moreover, Cronbach's alpha was 0.93 for the summary scale of the SRI. In the original German version, Cronbach's alpha ranged between 0.73 and 0.79 in three subscales and between 0.80 and 0.89 in four subscales, while it reached 0.89 for the summary scale [11]. This indicates that the reliability of the English SRI is even stronger than the original German version, which, however, is doubtlessly sufficient for use in group comparisons in the research setting [23].
The construct validity of the tool was obtained by factor analytical studies. Explorative factor analysis on the seven subscales using PCA revealed that three subscales loaded on two factors, while one factor could be extracted for the remaining four subscales. Therefore, two subscales could have been theoretically built out of one subscale for the three subscales loading on two factors. In addition, CFA revealed six subscales with two factor loadings; thus 12 subscales could have been theoretically built out of these six scales with the SRI consisting of 13 subscales when adding the remaining subscale with one factor loading according to CFA. As an example SRIAS, covered both daytime and nocturnal symptoms as reflected by two factors, and this is similar to the recent validation study on the SRI in COPD patients [12]. However, all factors were considerably correlated comparable to the recent validation in COPD patients. Therefore, the original seven-subscale structure was retained, as it was for the recent COPD validation.

Only one factor could be extracted out of the seven subscales for the summary scale of the English SRI, and this is comparable to the two validation studies on the original German SRI [10, 12]. Of note, this factor accounted for $70 \%$ of the total variance, but accounted for $59.8 \%$ (mixed cohort) and for $58.5 \%$ (COPD) of the total variance of the two German validation studies, respectively [10, 12]. For this reason, although high psychometric properties have been established for the original German SRI, the English SRI appears to be even superior. As a consequence, the presence of one summary scale has been established for the English SRI. Since the structure of the SRI contains social, psychological and physical components, this implies that these components cannot be reflected by independent summary scales in patients with severe chronic respiratory failure. This is in contrast to the SF-36, where both physical and mental health factors could be confirmed by factor analysis [4, 21].

The significant concurrent validity between the subscales of the SRI and the SF-36 highlighted the importance of a specific HRQL tool for patients with chronic respiratory failure. The highest correlation was found between the subscales focusing on comparable aspects of HRQL and the least correlation 


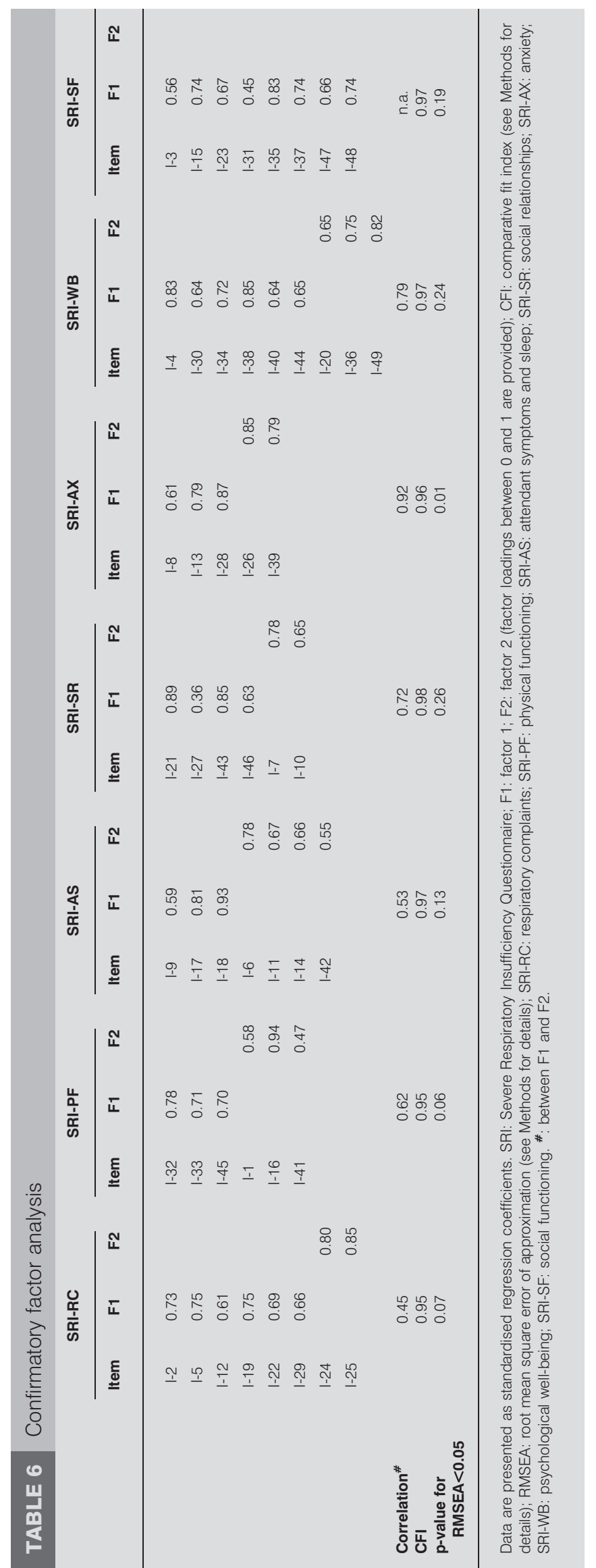

between the subscales which did not. This was comparable to the original validation $[25,26]$. SRI-SF strongly correlated with SF-36-VT. Similarly, SRI-WB correlated with SF-36-MH. There was a weak correlation between SRI-AS and SRI-RC with SF-36 scales. This was expected as the SF-36 was not designed to measure sleep disturbances or respiratory complaints, which are reportedly frequent in patients with chronic respiratory failure [25, 26]. SRI-SF showed a moderate-to-strong correlation with several SF-36 scales, indicating that a patient's ability to be involved in social activities is influenced by both physical and mental health factors.

Despite the given validity mentioned above, it has to be pointed out that the translation is formally valid for UK English only. In addition, the SRI is the result of professional forward and backward translation, but a weakness of this study is that this process was not formalised. Nevertheless, British and German researchers serving as authors of this study in addition to two independent native English translators have provided the UK English version of the SRI following a stepwise approach; thus a professional translation has been validated in the current trial. Furthermore, the authors would like to emphasise that for providing further validated translations of other languages, translators need to refer to the original German version and not to the translated English one.

Disease-associated anxiety is an important factor in HRQL in patients on long-term HMV. This is reflected by the scale SRIAX correlating moderately with SF-36-GH, SF-36-VT, SF-36-MH and SF-36-RE. During the development and validation of psychometric properties of SGRQ and the CRQ anxiety was attributed to psychological disturbances in patients with COPD $[6,27]$. Higher degree of anxiety has been shown to be related to higher degree of pulmonary impairment in patients with COPD [28]. Anxieties have been shown to improve after institution of HMV in patients with restrictive lung conditions, COPD and neuromuscular diseases [16, 24]. DUIVERMAN et al. [19] showed that in COPD the SRI total score strongly related to anxiety and depression as assessed by HADS (Hospital Anxiety and Depression Scale).

HRQL in patients with chronic respiratory failure is known to be significantly influenced by the underlying condition [11]. The construct validity is reinforced by the fact that COPD and non-COPD groups could be differentiated by administering SRI. The COPD patients had the worst score for SRI-SS and RCWD patients had the best HRQL, which is in keeping with previous studies with SRI [11, 18].

Previous prospective studies on impact of HMV in chronic hypercapnic COPD have shown conflicting results. Studies by ELLiotT et al. [29] and SivASOTHY et al. [30] did not show significant change in HRQL. However, MEECHAM JONES et al. [26] and PERRIN et al. [31] showed significant HRQL improvement. This difference might be attributed to the fact that different HRQL tools were used in these studies. Neither of these HRQL tools was specifically designed for patients on HMV. Recently, WINDISCH [16] has shown that specific HRQL as recorded by SRI significantly improved after instituting HMV and this was sustained at 1 yr. It was also shown that overall improvements were similar between COPD, restrictive chest wall diseases and neuromuscular diseases with 


\begin{tabular}{|c|c|c|c|c|c|c|c|c|}
\hline \multirow[t]{2}{*}{ TABLE 7} & \multicolumn{8}{|c|}{$\begin{array}{l}\text { Correlation analysis (Spearman) between the Severe Respiratory Insufficiency Questionnaire (SRI) and the Short-form } \\
\text { Health Survey (SF-36) subscales }\end{array}$} \\
\hline & SF-36-PF & SF-36-RP & SF-36-BP & SF-36-GH & SF-36-VT & SF-36-SF & SF-36-RE & SF-36-MH \\
\hline SRI-RC & 0.26 & 0.54 & 0.49 & 0.61 & 0.63 & 0.59 & 0.48 & 0.43 \\
\hline SRI-PF & 0.56 & 0.41 & 0.35 & 0.57 & 0.53 & 0.57 & 0.26 & 0.38 \\
\hline SRI-AS & 0.19 & 0.41 & 0.61 & 0.54 & 0.62 & 0.54 & 0.48 & 0.57 \\
\hline SRI-AX & 0.20 & 0.54 & 0.50 & 0.58 & 0.66 & 0.59 & 0.49 & 0.63 \\
\hline SRI-WB & 0.22 & 0.54 & 0.52 & 0.66 & 0.68 & 0.66 & 0.50 & 0.78 \\
\hline SRI-SF & 0.33 & 0.61 & 0.48 & 0.62 & 0.69 & 0.80 & 0.53 & 0.60 \\
\hline
\end{tabular}

hypercapnic respiratory failure [16]. Moreover, HRQL changes as assessed by the SRI have even been shown to be dependent on ventilatory strategy in COPD patients, with high-intensity NIV aimed at maximally improving blood gases being superior to the conventional approach of low-intensity NIV, which fails to improve blood gases [13]. This indicates that such a specific tool is better suited to studying this population and it is highly sensitive to change. This validation study paves the way for further studies in this area, especially in the large English-speaking populations using this disease-specific HRQL tool. Given the high psychometric properties of this version there is no reason to believe that this version would not be sensitive to change.

In conclusion, the English SRI is the result of a professional translation and back-translation of the well-validated original German SRI. The present validation study on the English SRI establishes high psychometric properties, which were even higher than in the original German validation study. Other adaptations of the SRI have shown similar high psychometric properties. Thereby, high internal consistency reliability as assessed by Cronbach's alpha coefficient, high construct validity as assessed by both explorative and confirmatory factor analysis, and high concurrent validity as assessed by the correlation analysis between SRI and SF-36 scales have been established. In addition, the English SRI is capable of differentiating between different conditions causing chronic respiratory failure. Other disease-specific questionnaires like CRQ or the SGRQ have not been validated in patients on HMV with varied underlying causes. They do not address all the aspects of daily life that might be affected by HMV and chronic respiratory failure [32]. The English SRI qualifies for being used in researches assessing health-related quality of life in patients with chronic respiratory failure on home ventilation.

\section{STATEMENT OF INTEREST}

A statement of interest for $\mathrm{W}$. Windisch can be found at www.erj. ersjournals.com/site/misc/statements.xhtml

\section{REFERENCES}

1 Higginson IJ, Carr AJ. Measuring quality of life: using quality of life measures in the clinical setting. BMJ 2001; 322: 1297-1300.
2 Testa MA, Simonson DC. Assesment of quality-of-life outcomes. N Engl J Med 1996; 334: 835-840.

3 Bergner M, Bobbitt RA, Carter WB, et al. The Sickness Impact Profile: development and final revision of a health status measure. Med Care 1981; 19: 787-805.

4 Ware JE Jr, Sherbourne CD. The MOS 36-item short-form health survey (SF-36). I. Conceptual framework and item selection. Med Care 1992; 30: 473-483.

5 Guyatt GH, Berman LB, Townsend M, et al. A measure of quality of life for clinical trials in chronic lung disease. Thorax 1987; 42: 773-778

6 Jones PW, Quirk FH, Baveystock CM. The St George's Respiratory Questionnaire. Respir Med 1991; 85: Suppl. B, 25-31.

7 Janssens JP, Penalosa B, Degive C, et al. Quality of life of patients under home mechanical ventilation for restrictive lung diseases: a comparative evaluation with COPD patients. Monaldi Arch Chest Dis 1996; 51: 178-184.

8 Elliott MW, Simonds AK, Carroll MP, et al. Domiciliary nocturnal nasal intermittent positive pressure ventilation in hypercapnic respiratory failure due to chronic obstructive lung disease: effects on sleep and quality of life. Thorax 1992; 47: 342-348.

9 Pehrsson K, Olofson J, Larsson S, et al. Quality of life of patients treated by home mechanical ventilation due to restrictive ventilatory disorders. Respir Med 1994; 88: 21-26.

10 Windisch W, Freidel K, Schucher B, et al. Evaluation of healthrelated quality of life using the MOS 36-Item Short-Form Health Status Survey in patients receiving noninvasive positive pressure ventilation. Intensive Care Med 2003; 29: 615-621.

11 Windisch W, Freidel K, Schucher B, et al. The Severe Respiratory Insufficiency (SRI) Questionnaire: a specific measure of healthrelated quality of life in patients receiving home mechanical ventilation. J Clin Epidemiol 2003; 56: 752-759.

12 Windisch W, Budweiser S, Heinemann F, et al. The Severe Respiratory Insufficiency Questionnaire was valid for COPD patients with severe chronic respiratory failure. J Clin Epidemiol 2008; 61: 848-853.

13 Dreher M, Storre JH, Schmoor C, et al. High-intensity versus lowintensity non-invasive ventilation in patients with stable hypercapnic COPD: a randomised crossover trial. Thorax 2010; 65: 303-308.

14 Storre JH, Seuthe B, Fiechter R, et al. Average volume-assured pressure support in obesity hypoventilation: a randomized crossover trial. Chest 2006; 130: 815-821.

15 Windisch W, Dreher M, Storre JH, et al. Nocturnal non-invasive positive pressure ventilation: physiological effects on spontaneous breathing. Respir Physiol Neurobiol 2006; 150: 251-260. 
16 Windisch W. Impact of home mechanical ventilation on healthrelated quality of life. Eur Respir J 2008; 32: 1328-1336.

17 Lopez-Campos JL, Failde I, Leon JA, et al. Calidad de vida relacionada con la salud de pacientes en programa de ventilación mecánica domiciliaria. La versión española del cuestionario SRI Health-related quality of life of patients receiving home mechanical ventilation: the Spanish version of the severe respiratory insufficiency questionnaire. Arch Bronconeumol 2006; 42: 588-593.

18 Lopez-Campos JL, Failde I, Masa JF, et al. Transculturally adapted Spanish SRI questionnaire for home mechanically ventilated patients was viable, valid, and reliable. J Clin Epidemiol 2008; 61: 1061-1066.

19 Duiverman ML, Wempe JB, Bladder G, et al. Health-related quality of life in COPD patients with chronic respiratory failure. Eur Respir J 2008; 32: 379-386.

20 Duiverman ML, Wempe JB, Bladder G, et al. Nocturnal noninvasive ventilation in addition to rehabilitation in hypercapnic patients with COPD. Thorax 2008; 63: 1052-1057.

21 Ghosh D, Rzehak P, Elliott MW, et al. Quality of life in home ventilation (HMV): validation of the English version of severe respiratory insufficiency (SRI) questionnaire. Eur Respir J 2010; 36: Suppl. 54, 701s.

22 Ware JE Jr. SF-36 Health Survey. Manual and Interpretation Guide. Boston, The Health Institute, New England Medical Center Hospitals, 1993.

23 Nunnally J, Bemstein I. Psychometric Theory. New York, McGraw Hill, 1994.

24 Mplus Statistical Analysis with Latent Variables, User's Guide. Version 4. Los Angeles, 2006.
25 Bach JR, Alba AS. Management of chronic alveolar hypoventilation by nasal ventilation. Chest 1990; 97: 52-57.

26 Meecham Jones DJ, Paul EA, Jones PW, et al. Nasal pressure support ventilation plus oxygen compared with oxygen therapy alone in hypercapnic COPD. Am J Respir Crit Care Med 1995; 152: 538-544.

27 Wijkstra PJ, TenVergert EM, Van Altena R, et al. Reliability and validity of the chronic respiratory questionnaire (CRQ). Thorax 1994; 49: 465-467.

28 Jones PW, Brusselle G, Dal Negro RW, et al. Health-related quality of life in patients by COPD severity within primary care in Europe. Respir Med 2011; 105: 57-66.

29 Elliott MW, Simonds AK, Carroll MP, et al. Domiciliary nocturnal nasal intermittent positive pressure ventilation in hypercapnic respiratory failure due to chronic obstructive lung disease: effects on sleep and quality of life. Thorax 1992; 47: 342-348.

30 Sivasothy P, Smith IE, Shneerson JM. Mask intermittent positive pressure ventilation in chronic hypercapnic respiratory failure due to chronic obstructive pulmonary disease. Eur Respir J 1998; 11: 34-40.

31 Perrin C, El Far Y, Vandenbos F, et al. Domiciliary nasal intermittent positive pressure ventilation in severe COPD: effects on lung function and quality of life. Eur Respir J 1997; 10: 2835-2839.

32 Carone M, Bertolotti G, Anchisi F, et al. Analysis of factors that characterize health impairment in patients with chronic respiratory failure. Quality of Life in Chronic Respiratory Failure Group. Eur Respir J 1999; 13: 1293-1300. 